ARTICLE OPEN

\title{
A putative enoyl-CoA hydratase contributes to biofilm formation and the antibiotic tolerance of Achromobacter
}

\author{
xylosoxidans
}

\author{
Lydia C. Cameron ${ }^{1}$, Benjamin Bonis ${ }^{1}$, Chi Q. Phan ${ }^{1,2}$, Leslie A. Kent ${ }^{1}$, Alysha K. Lee ${ }^{1,3}$ and Ryan C. Hunter (D)
}

\begin{abstract}
Achromobacter xylosoxidans has attracted increasing attention as an emerging pathogen in patients with cystic fibrosis. Intrinsic resistance to several classes of antimicrobials and the ability to form robust biofilms in vivo contribute to the clinical manifestations of persistent $A$. xylosoxidans infection. Still, much of $A$. xylosoxidans biofilm formation remains uncharacterized due to the scarcity of existing genetic tools. Here we demonstrate a promising genetic system for use in A. xylosoxidans; generating a transposon mutant library which was then used to identify genes involved in biofilm development in vitro. We further described the effects of one of the genes found in the mutagenesis screen, encoding a putative enoyl-CoA hydratase, on biofilm structure and tolerance to antimicrobials. Through additional analysis, we find that a fatty acid signaling compound is essential to A. xylosoxidans biofilm ultrastructure and maintenance. This work describes methods for the genetic manipulation of $A$. xylosoxidans and demonstrated their use to improve our understanding of $A$. xylosoxidans pathophysiology.
\end{abstract}

npj Biofilms and Microbiomes (2019)5:20; https://doi.org/10.1038/s41522-019-0093-6

\section{INTRODUCTION}

Cystic fibrosis (CF) is caused by mutations in the gene encoding the cystic fibrosis transmembrane conductance regulator (CFTR) protein that resides at the apical surface of many epithelial cell types. CFTR defects result in abnormal chloride and bicarbonate transport, increasing mucus viscosity in the pancreas, paranasal sinuses, digestive tract, and most notably, the lower airways. Viscous mucus, due to its impaired clearance and abundant nutrient bioavailability, in turn becomes chronically colonized by pathogenic bacteria that are the leading cause of mortality among the CF population. ${ }^{1}$ For decades, Pseudomonas aeruginosa and Staphylococcus aureus have been extensively characterized and recognized as the primary airway pathogens. More recently, however, many other multidrug-resistant opportunistic bacterial species have received attention for their association with CF disease progression. ${ }^{2}$

Among them, Achromobacter xylosoxidans is notable given its association with poor pulmonary function scores and apparent patient-to-patient transmissibility. ${ }^{3-5}$ This aerobic, Gram-negative opportunistic pathogen has been found to colonize anywhere from 2 to 20 percent of CF subjects, though its prevalence has risen in recent years, ${ }^{4,6-11}$ sparking a renewed interest in its pathophysiology. Of particular concern is the intrinsic and acquired resistance of $A$. xlyosoxidans to multiple classes of antimicrobial agents, including aminoglycosides, beta-lactams, carbapenems, chloramphenicol and fluoroquinolones, which presents a significant burden for infection control. ${ }^{7,8,12-15}$

Also complicating treatment of $A$. xylosoxidans is its ability to form robust biofilms. ${ }^{16-18}$ Thought to be the predominant in vivo lifestyle among CF pathogens, ${ }^{19}$ biofilms confer a highly protective growth environment that shields pathogens against environmental stress, antimicrobials and the host immune response. ${ }^{20}$ In addition, nutrient and oxygen gradients throughout microcolonies (or aggregates) ${ }^{16}$ result in slowed metabolism among biofilm cells which can also potentiate drug tolerance. The recalcitrance of biofilm cells to antibiotic exposure likely enhances the persistence of $A$. xylosoxidans during chronic infection of the CF lung.

Relative to canonical CF pathogens, comparatively little is known about the molecular mechanisms of biofilm formation and maintenance in A. xylosoxidans. This is due, in part, to the paucity of genetic tools available for manipulation of its genome. Therefore, the objectives of this study were two-fold: First, we sought to develop a tractable system to genetically manipulate A. xylosoxidans. Using this system, our second objective was to take a transposon mutagenesis approach to study the molecular basis of $A$. xylosoxidans biofilm formation. In doing so, we identified several gene products essential for biofilm development. We chose to further characterize a gene identified most frequently in our screen (echA) encoding a putative enoyl-CoA hydratase that, when disrupted, leads to a decrease in biofilm accumulation and increased susceptibility to multiple classes of antibiotics.

\section{RESULTS}

Identification of biofilm-defective mutants via transposon mutagenesis

To identify genetic determinants of biofilm formation in A. xylosoxidans, we generated a mutant library of strain MN001 using random transposon mutagenesis employing the minimariner transposable element. ${ }^{21}$ From this library, 15,000 mutants were screened for altered biofilm formation using an established

\footnotetext{
${ }^{1}$ Department of Microbiology \& Immunology, University of Minnesota, 689 23rd Avenue SE, Minneapolis, MN 55455, USA; ${ }^{2}$ Present address: Department of Genome Sciences, University of Washington, 3720 15th Ave NE, Seattle, WA 98195, USA; ${ }^{3}$ Present address: Department of Earth System Science, Stanford University, Stanford, CA 94305, USA Correspondence: Ryan C. Hunter (rchunter@umn.edu)
}

Received: 28 April 2019 Accepted: 17 July 2019

Published online: 06 August 2019 


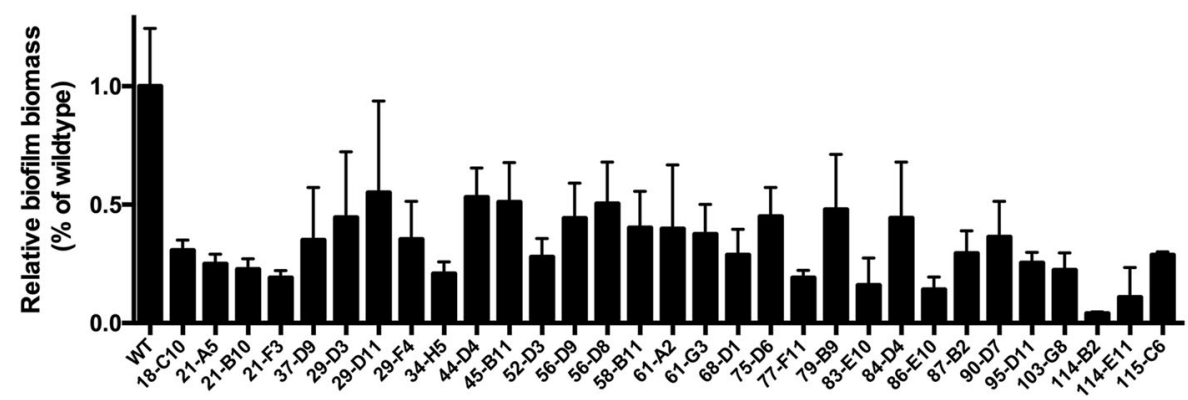

Fig. 1 Biofilm formation by transposon mutants of $A$. xylosoxidans MN001. Mean crystal violet absorbance for each mutant is expressed relative to the parental wildtype strain. Error bars represent standard deviation $(n=4)$

crystal violet (CV)/microtiter dish assay. ${ }^{22}$ After an initial round of screening, 134 putative biofilm-defective mutants were identified that were then re-tested $(n=4)$ to verify mutant phenotypes. After secondary screening and eliminating mutants with general growth defects, 31 transposon mutants were confirmed to be defective in biofilm accumulation (Fig. 1, Table 1).

To identify DNA sequences ( 200-400 bp) flanking transposon insertion junctions, mutants were screened by arbitrarily primed PCR. Insertions were mapped to loci predicted to encode various classes of gene products, including membrane proteins, glycosyltransferases, flagella, transcriptional regulators, in addition to several proteins with no assigned function (Table 1). Arbitrary PCR of three transposon insertion loci generated sequences with no homology to published gene sequences, and we were unable to obtain transposon-flanking sequences for five additional mutants. Of note, a putative enoyl-CoA hydratase, an exonuclease, and a propionate hydroxylase were each hit multiple times. We took interest in the gene encoding a putative enoyl-CoA hydratase (Axylo_0405) given the importance of homologous proteins in fatty acid signal biosynthesis and biofilm development among diverse bacterial species. ${ }^{23-29}$ We named this gene echA (for enoylCoA hydratase A) and sought to characterize its effects on $A$. xylosoxidans biofilm physiology in greater detail.

\section{Generation and complementation of an echA mutant}

Genetic tractability has been challenging for $A$. xylosoxidans due its inherent resistance to multiple classes of antibiotics and inducible efflux system as a second line of defense. ${ }^{12,13,15} \mathrm{We}$ therefore sought to develop a robust genetic system for the generation and complementation of marker-free deletion mutants that would facilitate further study of the genetic basis of biofilm formation. Given that tetracycline resistance was an effective marker to generate the transposon mutant library, the tetracycline resistance cassette from $\mathrm{pEX} 18 \mathrm{tc} \mathrm{c}^{30}$ was PCR amplified and ligated into the mobilizable suicide vector $\mathrm{pSMV} 8^{31}$ generating $\mathrm{pBMB} 1$ (empty vector) and pBMB2 (echA deletion construct; Supplementary Fig. 1). Attempts to construct a complementation vector using broad host-range cloning plasmids were unsuccessful. We therefore introduced echA into pBMB3 (pBBR1MCS-5::alcA) forming pBMB4 (Supplementary Fig. 1), placing echA expression under the control of an alcohol-inducible promoter. ${ }^{32}$ Using these vectors, we generated a markerless deletion mutant and complement of $A$. xylosoxidans MN001 lacking echA.

A. xylosoxidans biofilm ultrastructure is mediated by echA

To confirm that the biofilm-deficient phenotypes of transposon mutants 21-A5, 21-F3, and 86-E10 (Table 1) were a result of mutations in echA, we re-assessed biofilm formation by the markerless $\triangle$ echA mutant using the $\mathrm{CV}$ assay described above. As before, $\triangle e c h A$ exhibited a significant decrease in biofilm biomass accumulation relative to the wildtype ( $p=0.002)$ after $72 \mathrm{~h}$ (Fig. 2). Complementation with pBMB4 restored the wildtype phenotype
Table 1. List of independent biofilm-defective transposon mutants identified in this study

\begin{tabular}{|c|c|c|c|}
\hline Mutant & $\begin{array}{l}\text { Axylo } \\
\text { Locus Tag }\end{array}$ & Annotation & $\begin{array}{l}\text { Insertion } \\
\text { coordinate }\end{array}$ \\
\hline $18-\mathrm{C} 10$ & 2959 & $\begin{array}{l}\text { Extracellular serine protease } \\
\text { precursor }\end{array}$ & 3300783 \\
\hline $21-A 5$ & 0405 & Enoyl-CoA hydratase & 458026 \\
\hline 21-B10 & 2974 & DoxX family protein & 3318225 \\
\hline $21-\mathrm{F} 3$ & 0405 & Enoyl-CoA hydratase & 458968 \\
\hline 37-D9 & & No sequence & \\
\hline 29-D11 & 1017 & $\begin{array}{l}\text { Branched chain amino acid } \\
\text { transport protein }\end{array}$ & 1140377 \\
\hline 29-F4 & 3436 & $\begin{array}{l}\text { Flagellar basal body rod } \\
\text { protein FlgB }\end{array}$ & 3820439 \\
\hline 34-H5 & 1568 & Carboxymethylenebutenolidase & 1767696 \\
\hline 44-D4 & & No sequence & \\
\hline 45-B11 & 1417 & $\begin{array}{l}\text { Outer membrane protein } \\
\text { assembly factor BamD }\end{array}$ & 1595377 \\
\hline 52-D3 & 2959 & $\begin{array}{l}\text { Extracellular serine protease } \\
\text { precursor }\end{array}$ & 3296785 \\
\hline $56-\mathrm{D} 9$ & & No sequence & \\
\hline $61-A 2$ & 4754 & Hypothetical protein & 5265710 \\
\hline $61-G 3$ & 0328 & Hypothetical protein & 374132 \\
\hline 68-D1 & 2959 & $\begin{array}{l}\text { Extracellular serine protease } \\
\text { precursor }\end{array}$ & 3303694 \\
\hline 75-D6 & 0867 & Propionate hydroxylase & 961203 \\
\hline 77-F11 & 2959 & $\begin{array}{l}\text { Extracellular serine protease } \\
\text { precursor }\end{array}$ & 3302134 \\
\hline 79-B9 & 0165 & Glycosyltransferase CsbB & 200048 \\
\hline 83-E10 & 0126 & Hypothetical protein & 152964 \\
\hline 84-D4 & 0867 & Propionate hydroxylase & 961404 \\
\hline 86-E10 & 0405 & Enoyl-CoA hydratase & 458180 \\
\hline 87-B2 & 3872 & Glycosyltransferase MshA & 4315184 \\
\hline 90-D7 & & No sequence & \\
\hline 95-D11 & 1003 & Hypothetical protein & 1124527 \\
\hline 103-G8 & 5298 & L-asparaginase & 5841569 \\
\hline 114-B2 & 0867 & Propionate hydroxylase & 961364 \\
\hline 114-E11 & & No sequence & \\
\hline 115-C6 & 0951 & $\begin{array}{l}\text { LysR family transcriptional } \\
\text { regulator DmIR }\end{array}$ & 1065410 \\
\hline
\end{tabular}

( $p=0.023)$, confirming a role of $e c h A$ and its gene product in $A$. xylosoxidans biofilm development.

Enoyl-CoA hydratases are central to the biosynthesis of a class of fatty acid signaling molecules, or diffusible signal factors (DSFs), 
that have been described in diverse bacterial species for their role in mediating virulence, motility and biofilm development. ${ }^{24-29,33,34}$ In $P$. aeruginosa, for example, the DSF cis-2-decenoic acid (cis-DA) is known to play a critical role in biofilm dispersion. ${ }^{24,35}$ Therefore, to test whether the observed biofilm impairment phenotype in $A$. xylosoxidans was also mediated by a DSF-like signaling mechanism, we exogenously added synthetic cis-DA $(310 \mathrm{nM})^{35}$ to the $\triangle e c h A$ mutant during biofilm development. Despite having no effect on the WT strain, addition of cis-DA to $\triangle e c h A$ resulted in a significant restoration of biofilm biomass relative to the untreated control $(p=0.024)$ (Fig. 2). These data suggest that biofilm formation in MN001 is directly mediated by a DSF-like metabolite generated by an enoyl-CoA hydratase.

Since the CV staining approach used in the initial mutant screen relies on a dye that stains not only cells, but all biomass adhering to the microtiter plate, we elected to use additional biofilm assays to further characterize the biofilm phenotype of $\triangle e c h A$, and whether disruption of the putative enoyl-CoA hydratase

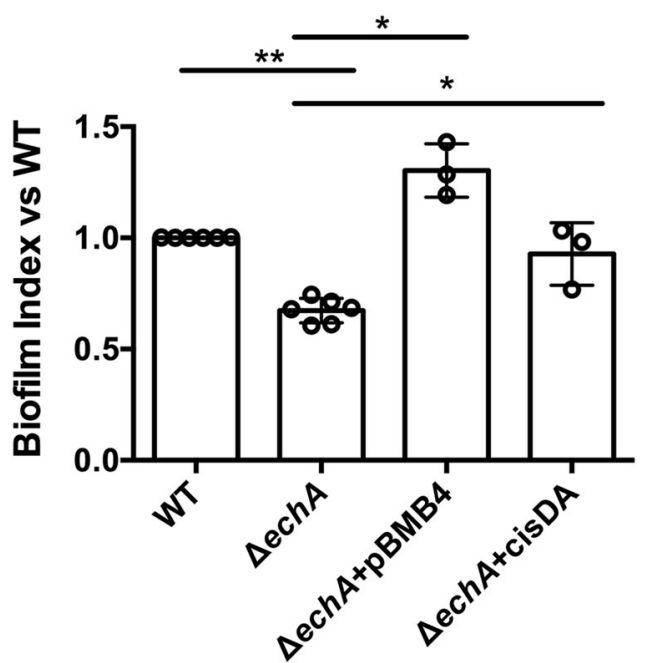

Fig. 2 A putative enoyl-CoA hydratase contributes to $A$. xylosoxidans biofilm formation via biosynthesis of a fatty acid signaling metabolite. A. xylosoxidans biofilms were grown for $72 \mathrm{~h}$, quantified using crystal violet and normalized to cell density (biofilm index). Deletion of echA resulted in a significant decrease in biofilm biomass that was restored via complementation and addition of cis-DA. Error bars represent standard error of the mean $(n=6$ for WT and $\Delta e c h A$; $n=3$ for $\Delta e c h A+$ pBMB4 and $\Delta e c h A+$ cis-DA) negatively impacts a specific stage of biofilm development (e.g. attachment, matrix production, maturation). We first used a modified adhesion assay ${ }^{36}$ to test for defects in attachment to the surface of a chamber slide. Under static conditions, $\triangle e c h A$ exhibited no difference in surface attachment relative to the wildtype, demonstrating that initial stages of biofilm formation are not affected (Fig. 3a). We then used a common colony morphology assay that relies on Congo red staining as a macroscopic means of identifying the capacity for extracellular matrix production. ${ }^{37,38}$ Over the course of 6 days of growth, biofilm colonies showed no apparent differences in morphology or color between strains, suggesting that the echA gene product has negligible effect on matrix production in $A$. xylosoxidans. Finally, we used scanning electron microscopy to visualize microcolony ultrastructure in mature biofilms grown for 72 hours under static conditions (Fig. 3c). Relative to WT, the $\triangle e c h A$ mutant exhibited notable phenotypic differences in biofilm architecture, corroborating observations made using the microtiter plate assay. First, the mutant strain demonstrated less robust biofilm growth and reduced surface coverage relative to WT. In addition, higher magnification images revealed a less-dense packing order to mutant biofilm cells, suggesting that cell-cell signaling mediated by enoyl-CoA hydratase-derived metabolite is central to biofilm ultrastructural development in A. xylosoxidans.

$\triangle e c h A$ exhibits increased susceptibility to antibiotic treatment Biofilm-associated bacteria can be orders of magnitude more tolerant to antibacterial compounds than their planktonic counterparts. ${ }^{20}$ Given its defect in biofilm formation and more diffuse nature of mature microcolonies shown by SEM, we hypothesized that the $\triangle e c h A$ mutant would exhibit increased susceptibility to antimicrobial treatment. To test this, mature biofilms were grown statically in 8-chamber coverslip slides for $72 \mathrm{~h}$ and treated with various concentrations of two commonly used therapeutics to which $A$. xylosoxidans is tolerant, levofloxacin and tobramycin (Fig. 4). Despite not showing any difference in minimum inhibitory concentrations for planktonic cells of the WT and mutant strains (100 $\mu \mathrm{g} / \mathrm{mL}$ for tobramycin; $4 \mu \mathrm{g} / \mathrm{mL}$ for levofloxacin), Live/Dead staining of treated biofilms, visualization by microscopy (Fig. 4a), and quantification of dead biomass (Fig. 4 b) revealed a statistically significant increase $(p<0.001)$ in susceptibility of the $\triangle e c h A$ biofilm to both antibiotics relative to WT. These data demonstrate a central role of echA in biofilm antibiotic tolerance.
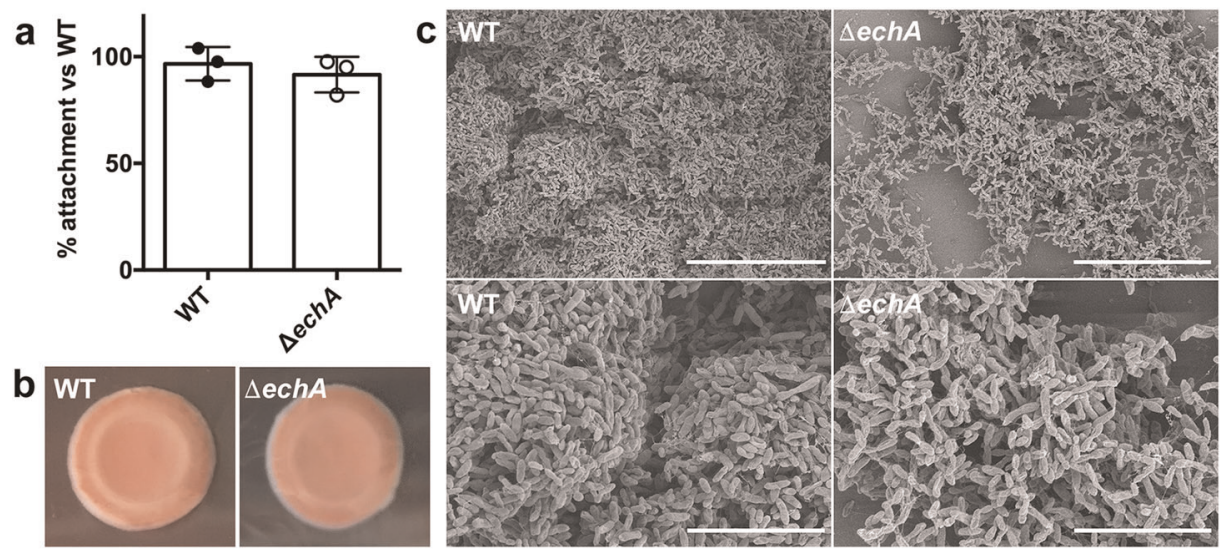

Fig. 3 echA plays a central role in biofilm ultrastructure in A. xylosoxidans MN001. a Early attachment assay for WT and echA mutant. Error bars represent standard deviation of the mean for replicate experiments $(n=3)$. b Colony biofilms grown on Congo red plates for 6 days revealed no differences in matrix production. c Mature biofilms of the WT and mutant were visualized by SEM to examine biofilm architecture (scale bars, top $=30 \mu \mathrm{m}$, bottom $=10 \mu \mathrm{m})$. All images shown are representative of experiments performed in biological triplicate $(n=3)$ with similar results 

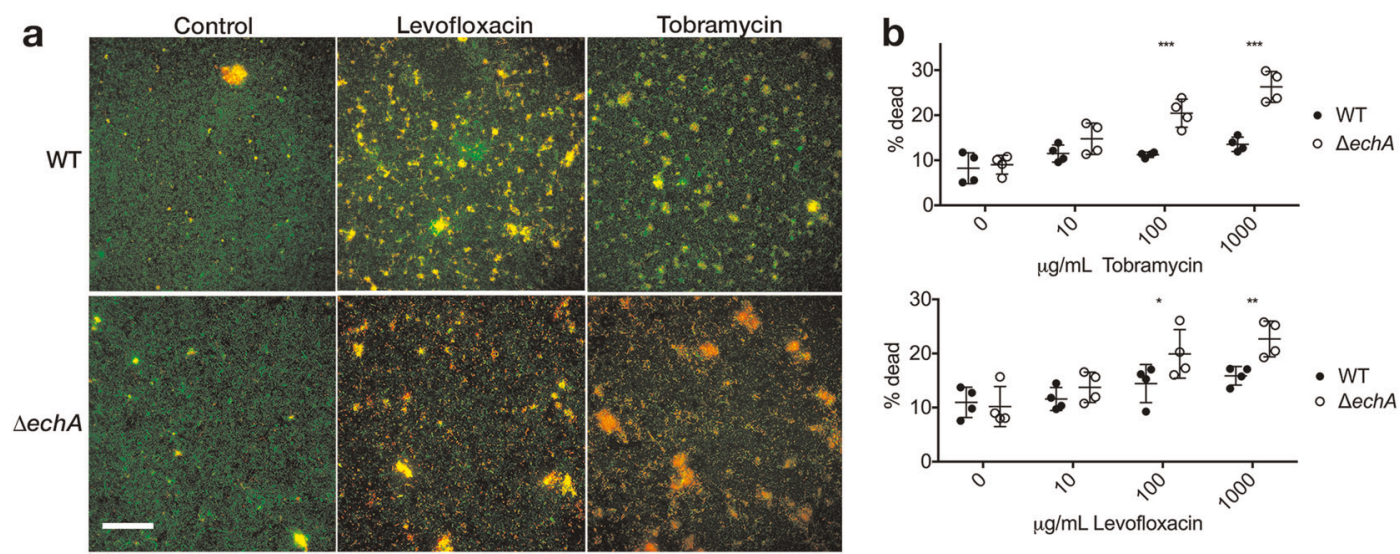

Fig. 4 Disruption of enoyl-CoA hydratase activity leads to increased antibiotic susceptibility. a Mature $A$. xylosoxidans biofilms were visualized by fluorescence microscopy after $6 \mathrm{~h}$ exposure to levofloxacin $(100 \mu \mathrm{g} / \mathrm{mL})$ and tobramycin $(1000 \mu \mathrm{g} / \mathrm{mL})$. Deletion of echA resulted in increased susceptibility to both compounds. $\mathbf{b}$ Integrative densities of green (live) and red (dead) cells were used to calculate \% dead biomass for each biofilm treated with various concentrations of antibiotic (bars represent standard deviation, $n=4$ for each treatment)

\section{DISCUSSION}

A. xylosoxidans is recognized as an emerging nosocomial pathogen and is associated with a range of infections, including bacteremia $^{39}$ endocarditis, ${ }^{40}$ meningitis, ${ }^{41}$ and pneumonia in immunocompromised individuals. ${ }^{42}$ In addition, the prevalence of $A$. xylosoxidans is now estimated to be as high as $20 \%$ among individuals with $\mathrm{CF}^{6-11}$ which is of increasing concern given its reported correlation to lung function decline, ${ }^{3}$ patient-to-patient transmissibility, ${ }^{5}$ and multi-drug resistance phenotypes. ${ }^{12-15}$ Unfortunately, little is known about the physiology and molecular biology of this pathogen, and a greater understanding is critically needed to inform new treatment strategies. This study is an important step in that direction as it identifies several molecular determinants of biofilm growth, thought to play a critical role in the persistence and pathogenesis of $A$. xylosoxidans in the CF airways. $^{16}$

The inherent and acquired drug resistance of Achromobacter spp. not only limits therapeutic approaches but has also led to a paucity of tools (i.e. selection markers) available for molecular biology studies of $A$. xylosoxidans and manipulation of its genome. Here, we systematically screened numerous vectors and determined that among antibiotics tested, only tetracycline and gentamicin resistance markers were compatible with both $E$. coli and A. xylosoxidans. Tet ${ }^{R}$ and $\mathrm{Gent}^{\mathrm{R}}$ cassettes were then used to generate (to our knowledge) the first transposon mutant library and a null deletion strain in A. xylosoxidans, facilitating a detailed study at the molecular level. These tools pave the way for addressing critical questions about the physiology of this bacterium and its role in pathogenesis.

Screening of our transposon library revealed several genetic determinants of biofilm formation. Statistically, genome coverage in our library was unsaturated ( 92\%), though 31 hits in genes in diverse functional categories suggests that the regulation of biofilm development in $A$. xylosoxidans integrates a complex network of cellular processes and environmental stimuli. Several homologs of genes identified in this screen have been linked to biofilm formation in other bacteria. For example, $f \lg B$, encoding a flagellar basal body rod protein, has been implicated in biofilm development in both Bordetella bronchiseptica ${ }^{43}$ and $P$. aeruginosa. ${ }^{44}$ It is noteworthy that other flagellar-associated genes are downregulated in $A$. xylosoxidans biofilms relative to planktonically grown cells. ${ }^{45}$ In addition, LysR family regulators, such as Axylo_0951 that was identified in our screen, have been implicated in biofilm morphology and mucosal colonization in other respiratory pathogens such as $P$. aeruginosa, Burkholderia cenocepacia, and Klebsiella pneumoniae. ${ }^{46-48}$ Further characterization of these and other genes identified in our screen (see Table 1) will undoubtedly generate new insights into the many aspects of Achromobacter biofilm physiology that remain uncharacterized.

We took most interest in Axylo_0405 (echA), encoding a putative enoyl-CoA hydratase (one of eight in strain MN001) with homology to Dspl in P. aeruginosa. ${ }^{24}$ Dspl and other bacterial homologs (e.g. RpfF in $X$. campestris) ${ }^{27}$ are key enzymes used in the synthesis of diffusible signaling factors (DSFs) that are monounsaturated fatty acids of medium chain length containing a cis-2 double bond thought to be central in their mechanism of action. These metabolites broadly regulate bacterial behaviors such as motility, iron uptake and virulence, ${ }^{29,49,50}$ and data presented here adds to the growing appreciation of their widespread role in biofilm development across diverse Gramnegative bacteria. While synthetic cis-DA was able to partially rescue the biofilm defect in $\triangle e c h A$, the specific identity of the fatty acid signal produced by $A$. xylosoxidans remains to be determined. It is also possible that $A$. xylosoxidans may respond to multiple DSF-like signals produced endogenously or exogenously, as interspecies signaling mediated by cis- 2 fatty acids has also been reported. ${ }^{28}$ Ongoing work is now aimed at characterizing the signal structure, its biosynthetic pathway, sensing mechanism(s) and downstream phenotypic effects in addition to biofilm development.

Respiratory pathogens that adopt a biofilm lifestyle in vivo exist in a protective environment against antimicrobials and host defense mechanisms. Furthermore, biofilm cells generally reduce their metabolic activity relative to planktonic cells, further enhancing their intrinsic resistance to therapy. A. xylosoxidans has been shown to form robust biofilms both in vivo and in vitro, ${ }^{16,18}$ which is corroborated by microscopy data presented here. However, our data demonstrating a heightened susceptibility of $\triangle e c h A$ to antibiotic treatment suggest that interfering with fatty acid-mediated cell-cell signaling may represent a viable approach to managing $A$. xylosoxidans biofilms in the lower airways, particularly when used in combination with conventional CF antibiotics. Here we elected to test two compounds to which $A$. xylosoxidans has high resistance, levofloxacin and tobramycin, ${ }^{51}$ though it will be interesting to test whether this effect holds true for other antibiotics with different mechanisms of action.

In summary, we developed a robust genetic system for use in A. xylosoxidans that we leveraged to generate a transposon mutant library. Further study of this library revealed a suite of genes essential for biofilm development in vitro and invoked an essential role for a putative enoyl-CoA hydratase in biofilm ultrastructure and tolerance to antimicrobials, likely mediated by a 
cis-2 fatty acid signaling compound. While a clear picture of the clinical impact of $A$. xylosoxidans in CF airway disease is only beginning to emerge, the continued study of the molecular basis of its biofilm formation and physiology will undoubtedly lead to improved treatment strategies for this important respiratory pathogen.

\section{METHODS}

Bacterial strains, plasmids, and growth conditions

Bacterial strains used are listed in Supplementary Table 1. A. xylosoxidans MNO01 $1^{52}$ and Escherichia coli were grown in lysogeny broth (LB) at $37^{\circ} \mathrm{C}$ unless otherwise specified. When necessary, growth media were supplemented with gentamicin at $20 \mu \mathrm{g} / \mathrm{ml}$ (E. coli) or $100 \mu \mathrm{g} / \mathrm{ml}$ (A. xlyosoxidans), ampicillin at $100 \mu \mathrm{g} / \mathrm{ml}$ (E. coli) or $300 \mu \mathrm{g} / \mathrm{ml}$ (A. xylosoxidans), carbenicillin $(300 \mu \mathrm{g} / \mathrm{ml})$, tetracycline $(300 \mu \mathrm{g} / \mathrm{ml})$, or chloramphenicol $(30 \mu \mathrm{g} / \mathrm{ml})$. E. coli strain $\beta 2155$ was supplemented with $360 \mu \mathrm{M}$ diaminopimelic acid (DAP).

\section{Transposon mutagenesis}

The transposon delivery vector pTnTet containing the hyperactive mariner transposon ${ }^{21}$ was introduced by transformation into the $E$. coli donor bacterial strain $\beta 2155 .{ }^{53}$ A. xylosoxidans MN001 and E. coli $\beta 2155$ (carrying pTnTet) were grown overnight in LB and LB containing chloramphenicol $\left(30 \mathrm{mg} / \mathrm{ml}\right.$ ) and $360 \mu \mathrm{M}$ DAP, respectively, at $37^{\circ} \mathrm{C}$. Cells were combined in a donor-to-recipient ratio of $5: 1$, centrifuged at $4000 \times g$ for $5 \mathrm{~min}$, resuspended in $200 \mu \mathrm{L}$ fresh LB-DAP and spot-plated $(10 \mu \mathrm{L})$ onto LB agar. Mating proceeded for $8 \mathrm{~h}$ at $37^{\circ} \mathrm{C}$, at which point cells were harvested and resuspended in $1 \mathrm{~mL}$ of LB. Cell suspensions were diluted 1:5, and $100 \mu \mathrm{L}$ aliquots were plated on LB-tetracycline agar and allowed to grow for $48 \mathrm{~h}$ at $37^{\circ} \mathrm{C}$. Colonies were randomly selected for downstream biofilm assays.

\section{Biofilm microtiter plate assay}

To identify determinants of biofilm development, transposon mutants were screened using a modified crystal violet (CV) assay approach. ${ }^{22}$ Briefly, individual mutants were transferred to single wells of a 96-well microtiter plate containing $200 \mu \mathrm{L}$ LB per well, and incubated while shaking at $37^{\circ} \mathrm{C}$. Following $24 \mathrm{~h}$ of growth, $2 \mu \mathrm{L}$ was transferred to $198 \mu \mathrm{L}$ ABT medium $\left[15 \mathrm{mM}\left(\mathrm{NH}_{4}\right)_{2} \mathrm{SO}_{4}, 40 \mathrm{mM} \mathrm{Na}_{2} \mathrm{HPO}_{4}, 20 \mathrm{mM} \mathrm{KH}_{2} \mathrm{PO}_{4}, 50 \mathrm{mM}\right.$ $\mathrm{NaCl}, 1 \mathrm{mM} \mathrm{MgCl}, 0.1 \mathrm{mM} \mathrm{CaCl}$ and $0.01 \mathrm{mM} \mathrm{FeCl}_{3}$ supplemented with $0.5 \%$ casamino acids and $0.5 \%$ glucose $]^{25}$ in a new microtiter plate, and grown for an additional $24 \mathrm{~h}$ at $37^{\circ} \mathrm{C}$ in a humidified chamber. Plates were first measured spectrophotometrically $\left(\mathrm{OD}_{600}\right)$ to determine culture cell density. Supernatants were discarded, and plates were washed $3 \mathrm{X}$ with ultrapure water. Plates were dried in a biosafety hood for $2.5 \mathrm{~h}$ and stained with $200 \mu \mathrm{L}$ of $0.1 \% \mathrm{CV}$ for 20 minutes. Plates were then washed $5 \mathrm{X}$ to remove excess stain, air-dried for $4 \mathrm{~h}$, and $200 \mu \mathrm{L} 30 \%$ acetic acid was added to each well. Following a 15 min incubation, CV absorbance was measured spectrophotometrically $\left(\mathrm{OD}_{560}\right)$ and normalized to culture density. Wells exhibiting less than $50 \%$ absorbance than the wildtype (MN001) were considered putative hits (134 total) and were subjected to a secondary screen using the same protocol ( $n=4$ for each mutant). Transposon mutants showing a significant reduction in biofilm growth (31 total, as determined by a Mann-Whitney $U$ test) were stored for further characterization.

Biofilm growth of MN001, its $\triangle e c h A$ derivative and complemented strain (see below) were also tested using the same microtiter assay. In these experiments, growth medium was supplemented with $2 \%$ ethanol to drive expression of the alcA promoter in pBMB4 (below). When indicated, cis-2decenoic acid (F13807D, Carbosynth) was also added at a concentration of $310 \mathrm{nM}^{35}$

\section{Arbitrary PCR and mutant sequencing}

An arbitrary-PCR-based approach ${ }^{22}$ was used to identify sequences flanking transposon insertion sites. The PCR method involved two rounds of reactions, with the first using a primer unique for the mariner transposon and one degenerate primer (pair 1, Table S2). ${ }^{54}$ The second round included nested primers (pair 2) unique to the transposon and $5^{\prime}$ end of the arbitrary primer for amplification of PCR products obtained in the first round. PCR products were sequenced at the University of Minnesota Genomics Center (UMGC) and were mapped to the $A$. xylosoxidans MN001 genome (Accession\#PRJNA288995).
Construction and complementation of an echA deletion mutant

To generate a deletion vector compatible with $A$. xylosoxidans, pSMV8 $8^{55}$ was first digested using Apal. A tetracycline resistance cassette was then amplified from pEX18tc ${ }^{31}$ using primer pair 3 (Supplementary Table 2) and digested with Apal. Vector and insert (1:3 ratio) were then ligated using T4 ligase, transformed into E. coli UQ950 and selected for on LB agar containing tetracycline $(15 \mu \mathrm{g} / \mathrm{mL})$. Colonies were screened using primers M13F and TetR (pair 4) to confirm insertion orientation. One plasmid, pBMB1, was selected for further use.

To generate an in-frame, unmarked deletion of Axylo1_0405 (echA), $\sim 1 \mathrm{~kb}$ sequences flanking echA were PCR amplified using primer pairs 5 and 6 (Supplementary Table 2). These flanking regions were combined and cloned into pBMB1 digested with Spel and Xhol using Gibson assembly, resulting in pBMB2 (Supplementary Fig. 1). This plasmid was then chemically transformed into UQ950, and positive ligations were screened by PCR. pBMB2 was then transformed into E. coli strain WM3064 and mobilized into A. xylosoxidans MN001 by conjugation. Recombinants were selected for on LB-tetracycline agar and double recombinants were selected for on LB agar containing $6 \%$ sucrose.

Complementation was achieved via exogenous expression of echA (Ax_0405) from pBBR1MCS-5. ${ }^{55}$ To do so, an alcohol-inducible promoter, alc ${ }^{32}$ was first amplified from pGGA008 using primers alcA_F and alcA_R (Supplementary Table 2) before digestion with restriction enzymes HindllI and BamHI. Ligation into similarly digested pBBR1MCS-5 yielded pBMB3 (pBBR1MCS-5::alcA). echA was then amplified from A. xylosoxidans MN001 genomic DNA using primers echA_F and echA_R, and digested with BamHI and Sacl before ligation into $\mathrm{PBMB} 3$ using T4 ligase. The resulting vector, pBMB4 (pBBR1MCS-5::alcAechA; Supplementary Fig. 1), was transformed into $E$. coli UQ950. This vector was then introduced into $A$. xylosoxidans MN001 via conjugation with an E. coli donor strain WM3064, and transconjugants were selected on LB agar containing $300 \mu \mathrm{g} / \mathrm{mL}$ gentamicin sulfate. All constructs and positive transformants were verified by Sanger sequencing.

\section{Attachment assay}

A modified attachment assay ${ }^{36}$ was used to assess early attachment of A. xylosoxidans to a polystyrene substratum. Briefly, MN001 and its $\triangle e c h A$ derivative were grown for $18 \mathrm{~h}$ at $37^{\circ} \mathrm{C}$ in $\mathrm{ABT}$ followed by dilution 1:100 into fresh medium. Cells were then grown to mid-log phase $\left(\mathrm{OD}_{600}=0.6\right)$ before dilution in $\mathrm{ABT}$ to an $\mathrm{OD}_{600}$ of 0.1. $200 \mu \mathrm{l}$ of each culture was added to an 8-chamber coverslip slide (Ibidi, \#80824) and incubated at $37^{\circ} \mathrm{C}$ for $1 \mathrm{~h}$. Following incubation, slides were rinsed twice with $200 \mu \mathrm{l}$ of PBS to remove unattached biomass, and attached cells were stained using SYTO 9 (Invitrogen) in PBS according the manufacturer's protocol. Substrata were imaged using an Olympus IX83 inverted fluorescence microscope with a transmitted Koehler illuminator and a 40X objective lens (Olympus). Four images per strain per biological replicate $(n=4)$ were captured on a Hamamatsu ORCA camera, and post-acquisition analysis was performed using FIJI software ${ }^{56}$ by calculating the integrated density of SYTO 9.

\section{Colony biofilm assay}

MN001 and $\triangle$ echA were grown overnight in LB medium, diluted 1:1000, and $10 \mu \mathrm{L}$ was spotted on nutrient agar containing $1 \%$ tryptone, $1 \%$ agar, $20 \mu \mathrm{g} / \mathrm{mL}$ Coomassie Brilliant Blue, and $40 \mu \mathrm{g} / \mathrm{mL}$ Congo red. ${ }^{36}$ Plates were incubated at $37^{\circ} \mathrm{C}$ for 6 days and monitored daily for colony morphology.

\section{Scanning electron microscopy}

Overnight cultures were diluted 1:10 in fresh LB medium and were added to 48-well microtiter plates containing autoclaved Aclar fluoropolymer film (Electron Microscopy Sciences, Hatfield, PA). Biofilms were grown for $48 \mathrm{~h}$ at $37^{\circ} \mathrm{C}$, shaking at $50 \mathrm{rpm}$, and prepared for SEM using cationic dye stabilization methods. ${ }^{57,58}$ Briefly, Aclar membranes containing biofilm growth were washed three times in $0.2 \mathrm{M}$ sodium cacodylate buffer, and submerged in primary fixative $(0.15 \mathrm{M}$ sodium cacodylate buffer, $\mathrm{pH} 7.4$, $2 \%$ paraformaldehyde, $2 \%$ glutaraldehyde, $4 \%$ sucrose, $0.15 \%$ alcian blue 8 $\mathrm{GX}$ ) for $22 \mathrm{~h}$. Samples were washed three more times prior to a 90 minute treatment with secondary fixative ( $1 \%$ osmium tetroxide, $1.5 \%$ potassium ferrocyanide, $0.135 \mathrm{M}$ sodium cacodylate, $\mathrm{pH}$ 7.4). After three final washes, biofilms were chemically dehydrated in a graded ethanol series $(25,50,70$, $85,95[2 \times]$ and $100 \%[2 \times])$ before $\mathrm{CO}_{2}$-based critical point drying. Aclar membranes were attached to SEM specimen mounts using carbon conductive adhesive tape and sputter coated with $\sim 5 \mathrm{~nm}$ iridium using 
the Leica ACE 600 magnetron-based system. Biofilms were imaged using a Hitachi S-4700 field emission SEM with an operating voltage of $2 \mathrm{kV}$.

\section{Antibiotic challenge}

Biofilm antimicrobial susceptibility testing was performed using a chamber slide assay. ${ }^{59}$ Briefly, MN001 and $\triangle e c h A$ were grown in LB overnight, diluted 1:1000 and grown to an $\mathrm{OD}_{600}$ of 0.6 before dilution to an $\mathrm{OD}_{600}$ of 0.5 . In total $200 \mu \mathrm{L}$ of each culture was then added to each well of an Ibidi 8-chamber coverslip slide and incubated at $37^{\circ} \mathrm{C}$ in a humidified chamber. After $24 \mathrm{~h}$, medium was replaced with fresh LB and incubated for an additional $24 \mathrm{~h}$. Media was gently aspirated from each well, replaced with LB containing either tobramycin or levofloxacin $(0,10,100$, and $1000 \mu \mathrm{g} / \mathrm{mL})$, and incubated at $37^{\circ} \mathrm{C}$ for $6 \mathrm{~h}$. Cells were washed in sterile PBS to remove unattached biomass, stained for 15 minutes using the BacLight Live/Dead viability assay (Life Technologies, \#L7012) and visualized by fluorescent imaging as described above. Integrated density for SYTO 9 and propidium iodide (PI) for each image was determined using $\mathrm{FIJI}^{56}$ and percentage of dead biomass was determined by (average integrative density of $\mathrm{PI}$ )/(average integrated density of $\mathrm{PI}+$ integrated density of SYTO 9). ${ }^{59}$ Data were generated using four biological replicates $(n=4)$.

\section{Prior publication}

This work was previously published as a preprint. ${ }^{60}$

\section{Reporting Summary}

Further information on research design is available in the Nature Research Reporting Summary linked to this article.

\section{DATA AVAILABILITY}

All data generated during and/or analyzed during the current study are included in this article, its Supplementary information files, or are available from the corresponding author on reasonable request.

\section{ACKNOWLEDGEMENTS}

pGGA008 (Addgene plasmid \#48817) was a gift from Dr. Jan Lohmann (Heidelberg). We thank Clayton Summitt for assistance with data analysis and other members of the Hunter lab for critical review of the manuscript. Parts of this work were carried out in the Characterization Facility, University of Minnesota, which receives partial support from NSF through the Materials Research Science and Engineering Centers (MRSEC) program. This study was supported by the University of Minnesota, a NHLBI Pathway to Independence award to RCH, American Society for Microbiology Undergraduate Research Fellowships to AKL and LAK.

\section{AUTHOR CONTRIBUTIONS}

L.C. performed experiments, created figures, and helped write and edit the manuscript. B.B. performed experiments, created figures, and helped edit the manuscript. C.P. and A.L. performed experiments. L.K. performed SEM experiments and created figures. R.H. contributed to the conception, experimental design, data interpretation, statistical analyses, and manuscript preparation.

\section{ADDITIONAL INFORMATION}

Supplementary information accompanies the paper on the npj Biofilms and Microbiomes website (https://doi.org/10.1038/s41522-019-0093-6).

Competing interests: The authors declare no competing interests.

Publisher's note: Springer Nature remains neutral with regard to jurisdictional claims in published maps and institutional affiliations.

\section{REFERENCES}

1. Gibson, R. L., Burns, J. L. \& Ramsey, B. W. Pathophysiology and management of pulmonary infections in cystic fibrosis. Am. J. Respir. Crit. Care. Med. 168, 918-951 (2003).

2. Salsgiver, E. L. et al. Changing epidemiology of the respiratory bacteriology of patients with cystic fibrosis. Chest 149, 390-400 (2016).
3. Ridderberg, W., Bendstrup, K. E., Olesen, H. V., Jensen-Fangel, S. \& NørskovLauritsen, N. Marked increase in incidence of Achromobacter xylosoxidans infections caused by sporadic acquisition from the environment. J. Cyst. Fibros. 10, 466-469 (2011).

4. Rønne Hansen, C., Pressler, T., Høiby, N. \& Gormsen, M. Chronic infection with Achromobacter xylosoxidans in cystic fibrosis patients; a retrospective case control study. J. Cyst. Fibros. 5, 245-251 (2006).

5. Van Daele, S. et al. Shared genotypes of Achromobacter xylosoxidans strains isolated from patients at a cystic fibrosis rehabilitation center. J. Clin. Microbiol. 43, 2998-3002 (2005).

6. Burns, J. L. et al. Microbiology of sputum from patients at cystic fibrosis centers in the United States. Clin. Infect. Dis. 27, 158-163 (1998).

7. Tan, K., Conway, S. P., Brownlee, K. G., Etherington, C. \& Peckham, D. G. Alcaligenes infection in cystic fibrosis. Pediatr. Pulmonol. 34, 101-104 (2002).

8. Raso, T., Bianco, O., Grosso, B., Zucca, M. \& Savoia, D. Achromobacter xylosoxidans respiratory tract infections in cystic fibrosis patients. APMIS 116, 837-841 (2008).

9. Pereira, R. H. et al. Patterns of virulence factor expression and antimicrobial resistance in Achromobacter xylosoxidans and Achromobacter ruhlandii isolates from patients with cystic fibrosis. Epidemiol. Infect. 145, 600-606 (2017).

10. De Baets, F., Schelstraete, P., Van Daele, S., Haerynck, F. \& Vaneechoutte, M. Achromobacter xylosoxidans in cystic fibrosis: Prevalence and clinical relevance. J. Cyst. Fibros. 6, 75-78 (2007).

11. Amoureux, L. et al. Detection of Achromobacter xylosoxidans in hospital, domestic, and outdoor environmental samples and comparison with human clinical isolates. Appl. Environ. Microbiol. 79, 7142-7149 (2013).

12. Bador, J., Amoureux, L., Blanc, E. \& Neuwirth, C. Innate aminoglycoside resistance of Achromobacter xylosoxidans is due to axyXY-oprZ, an RND-type multidrug efflux pump. Antimicrob. Agents Chemother. 57, 603-605 (2013).

13. Bador, J. et al. First description of an RND-type multidrug efflux pump in Achromobacter xylosoxidans, AxyABM. Antimicrob. Agents Chemother. 55, 4912-4914 (2011)

14. Amoureux, L. et al. Epidemiology and resistance of Achromobacter xylosoxidans from cystic fibrosis patients in Dijon, Burgundy: First French data. J. Cyst. Fibros. 12, 170-176 (2013).

15. Rolston, K. V. \& Messer, M. The in-vitro susceptibility of Alcaligenes denitrificans subsp. xylosoxidans to 40 antimicrobial agents. J. Antimicrob. Chemother. 26, 857-860 (1990).

16. Nielsen, S. M., Nørskov-Lauritsen, N., Bjarnsholt, T. \& Meyer, R. L. Achromobacter species isolated from cystic fibrosis patients reveal distinctly different biofilm morphotypes. Microorganisms 4, pii: E33 (2016).

17. Hansen, C. R. et al. Inflammation in Achromobacter xylosoxidans infected cystic fibrosis patients. J. Cyst. Fibros. 9, 51-58 (2010).

18. Jakobsen, T. H. et al. Complete genome sequence of the cystic fibrosis pathogen Achromobacter xylosoxidans NH44784-1996 complies with important pathogenic phenotypes. PLoS ONE 8, e68484 (2013).

19. Bjarnsholt, T. et al. The in vivo biofilm. Trends Microbiol. 21, 466-474 (2013).

20. Stewart, P. S. \& Costerton, J. W. Antibiotic resistance of bacteria in biofilms. Lancet 358, 135-138 (2001).

21. Chiang, S. L. \& Rubin, E. J. Construction of a mariner-based transposon for epitope-tagging and genomic targeting. Gene 296, 179-185 (2002).

22. O'Toole, G. A. et al. Genetic approaches to study of biofilms. Methods Enzym. 310, 91-109 (1999).

23. Wang, L. H. et al. A bacterial cell-cell communication signal with cross-kingdom structural analogues. Mol. Microbiol. 51, 903-912 (2004).

24. Amari, D. T., Marques, C. N. H. \& Davies, D. G. The putative enoyl-coenzyme A hydratase Dspl is required for production of the Pseudomonas aeruginosa biofilm dispersion autoinducer cis-2-decenoic acid. J. Bacteriol. 195, 4600-4610 (2013).

25. Ionescu, M. et al. Promiscuous diffusible signal factor production and responsiveness of the Xylella fastidiosa Rpf system. mBio 7, e01054-16 (2016).

26. Newman, K. L., Almeida, R. P. P., Purcell, A. H. \& Lindow, S. E. Cell-cell signaling controls Xylella fastidiosa interactions with both insects and plants. Proc. Natl Acad. Sci. USA 101, 1737-1742 (2004).

27. Dow, J. M. et al. Biofilm dispersal in Xanthomonas campestris is controlled by cellcell signaling and is required for full virulence to plants. Proc. Natl Acad. Sci. USA 100, 10995-11000 (2003).

28. Boon, C. et al. A novel DSF-like signal from Burkholderia cenocepecia interferes with Candida albicans morphological transition. ISME J. 2, 27-36 (2003).

29. Huang, T. P. \& Lee Won, A. C. Extracellular fatty acids facilitate flagellaindependent translocation by Stenotrophomonas maltophila. Res. Microbiol. 158, 702-711 (2007).

30. Kovach, M. E. et al. Four new derivatives of the broad-host-range cloning vector pBBR1MCS, carrying different antibiotic-resistance cassettes. Gene 166, 175-176 (1995).

31. Hoang, T. T., Karkhoff-Schweizer, R. R., Kutchma, A. J. \& Schweizer, H. P. A broadhost-range Flp-FRT recombination system for site-specific excision of 
chromosomally-located DNA sequences: Application for isolation of unmarked Pseudomonas aeruginosa mutants. Gene 212, 77-86 (1998).

32. Lampropoulos, A. et al. Greengate-a novel, versatile, and efficient cloning system for plant transgenesis. PLoS ONE 8, e83043 (2013).

33. Deng, Y. et al. Cis-2-dodecenoic acid receptor RpfR links quorum-sensing signal perception with regulation of virulence through cyclic dimeric guanosine monophosphate turnover. Proc. Natl Acad. Sci. USA 109, 15479-15484 (2012).

34. Barber, C. E. et al. A novel regulatory system required for pathogenicity of Xanthomonas campestris is mediated by a small diffusible signal molecule. Mol. Microbiol. 24, 555-566 (1997).

35. Davies, D. G. \& Marques, C. N. H. A fatty acid messenger is responsible for inducing dispersion in microbial biofilms. J. Bacteriol. 191, 1393-1403 (2009).

36. Ramsey, M. M. \& Whiteley, M. Pseudomonas aeruginosa attachment and biofilm development in dynamic environments. Mol. Microbiol. 53, 1075-1087 (2004).

37. Dietrich, L. E. et al. Bacterial community morphogenesis is intimately linked to the intracellular redox state. J. Bacteriol. 195, 1371-1380 (2013).

38. Jones, C. J. \& Wozniak, D. J. Congo red stain identifies matrix overproduction and is an indirect measurement for c-di-GMP in many species of bacteria In Sauer K. (ed) c-di-GMP signaling. Meth. Mol. Biol. Humana Press, New York, NY (2017).

39. Aisenberg, G., Rolston, K. V. \& Safdar, A. Bacteremia caused by Achromobacter and Alcaligenes species in 46 patients with cancer. Cancer 101, 2134-2140 (2004).

40. Ahmed, M. S. et al. Achromobacter xylosoxidans, an emerging pathogen in catheter-related infection in dialysis population causing prosthetic valve endocarditis: a case report and review of literature. Clin. Nephrol. 71, 350-354 (2009).

41. Namnyak, S. S., Holmes, B. \& Fathalla, S. E. Neonatal meningitis caused by Achromobacter xylosoxidans. J. Clin. Microbiol. 22, 470-471 (1985),

42. Wood, G. C. et al. Treatment of Achromobacter ventilator-associated pneumonia in critically ill trauma patients. Ann. Pharmacother. 52, 120-125 (2018).

43. Nicholson, T. L., Conover, M. S. \& Deora, R. Transcriptome profiling reveals stagespecific production and requirement of flagella durting biofilm development in Bordetella bronchiseptica. PLoS ONE 7, e49166 (2012).

44. De La Fuente-Nunez, C. et al. Inhibition of bacterial biofilm formation and swarming motility by a small synthetic cationic peptide. Antimicrob Agents Chemother 56, 2696-2704 (2012).

45. Nielsen, S. M., Penstoft, L. N. \& Norskov-Lauritsen, N. Motility, biofilm formation and antimicrobial efflux of sessile and planktonic cells of Achromobacter xylosoxidans. Pathogens 8, 14 (2019).

46. Hennequin, C. \& Forestier, C. oxyR, a LysR-type regulator involved in Klebsiella pneumoniae mucosal and abiotic colonization. Infect. Immun. 77, 5449-5457 (2009)

47. Cao, $\mathrm{H}$. et al. A quorum sensing-associated virulence gene of Pseudomonas aeruginosa encodes a LysR-like transcription regulator with a unique self-regulatory mechanism. Proc. Natl Acad. Sci. USA 98, 14613-14618 (2001).

48. Bernier, S. P., Nguyen, D. T. \& Sokol, P. A. A LysR-type transcriptional regulator in Burkholderia cenocepacia influences colony morphology and virulence. Infect. Immun. 76, 38-47 (2008).

49. Huedo, P. et al. Two different $r p f$ clusters distributed among a population of Stenotrophomonas maltophila clinical strains display differential diffusible signal factor production and virulence regulation. J. Bacteriol. 196, 2431 (2014).
50. Chatterjee, S. \& Sonti, R. V. rpfF mutants of Xanthomonas oryzae sp. oryzae are deficient for virulence and growth under low iron conditions. Mol. Plant Microbe Interact. 15, 463-471 (2002).

51. Abbott, I. J. \& Peleg, A. Y. Stenotrophomonas, Achromobacter, and nonmelioid Burkholderia species: antimicrobial resistance and therapeutic strategies. Sem. Resp. Crit. Care Med. 36, 99-110 (2015).

52. Badalamenti, J. P. \& Hunter, R. C. Complete genome sequence of Achromobacter xylosoxidans MN001, a cystic fibrosis airway isolate. Genome Announc 3, pii: e00947-15 (2015)

53. Dehio, C. \& Meyer, M. Maintenance of broad-host-range incompatibility group $p$ and group q plasmids and transposition of tn5 in bartonella henselae following conjugal plasmid transfer from Escherichia coli. J. Bacteriol. 179, 538-540 (1997).

54. Larsen, R. A., Wilson, M. M., Guss, A. M. \& Metcalf, W. W. Genetic analysis of pigment biosynthesis in xanthobacter autotrophicus py2 using a new, highly efficient transposon mutagenesis system that is functional in a wide variety of bacteria. Arch. Microbiol. 178, 193-201 (2002).

55. Saltikov, C. W., Cifuentes, A., Venkateswaran, K. \& Newman, D. K. The ars detox ification system is advantageous but not required for $\mathrm{As}(\mathrm{v})$ respiration by the genetically tractable Shewanella species strain ANA-3. Appl. Environ. Microbiol. 69, 2800-2809 (2003).

56. Schindelin, J. et al. FIJl: An open-source platform for biological-image analysis. Nat. Methods 9, 676-682 (2012).

57. Erlandsen, S. L., Kristich, C. J., Dunny, G. M. \& Wells, C. L. High-resolution visualization of the microbial glycocalyx with low-voltage scanning electron microscopy: dependence on cationic dyes. J. Histochem. Cytochem. 52, 1427-1435 (2004)

58. Barnes, A. M., Ballering, K. S., Leibman, R. S., Wells, C. L. \& Dunny, G. M. Enterococcus faecalis produces abundant extracellular structures containing DNA in the absence of cell lysis during early biofilm formation. mBio 3, e00193-00112 (2012).

59. Tom, S. K., Yau, Y. C., Beaudoin, T., LiPuma, J. J. \& Waters, V. Effect of high-dose antimicrobials on biofilm growth of Achromobacter species isolated from cystic fibrosis patients. Antimicrob. Agents Chemother. 60, 650-652 (2016).

60. Cameron, L. C., et al. A putative enoyl-CoA hydratase contributes to biofilm formaton and the antibiotic tolerance of Achromobacter xylosoxidans. Preprint at http://www.biorxiv.org/content/10.1101/620559v1 (2019).

Open Access This article is licensed under a Creative Commons Attribution 4.0 International License, which permits use, sharing, adaptation, distribution and reproduction in any medium or format, as long as you give appropriate credit to the original author(s) and the source, provide a link to the Creative Commons license, and indicate if changes were made. The images or other third party material in this article are included in the article's Creative Commons license, unless indicated otherwise in a credit line to the material. If material is not included in the article's Creative Commons license and your intended use is not permitted by statutory regulation or exceeds the permitted use, you will need to obtain permission directly from the copyright holder. To view a copy of this license, visit http://creativecommons. org/licenses/by/4.0/.

(c) The Author(s) 2019 